\title{
The Potential Role of Wedololactone in Treating Particle-induced Osteolysis_A Mouse Calvarial Model
}

\section{Yung-Chang Lu}

Mackay Medical College

Ting-Kuo Chang

Mackay Medical College

Tzu-Chiao Lin

Mackay Memorial Hospital

Shu-Ting Yeh

Mackay Memorial Hospital

Hsu-Wei Fang

National Taipei University of Technology

Chun-Hsiung Huang

Changhua Christian Medical Foundation Changhua Christian Hospital

Chang-Hung Huang ( $\square$ changhung0812@gmail.com )

Mackay Memorial Hospital Tamshui Branch Hospital https://orcid.org/0000-0002-9936-0182

\section{Research}

Keywords: Particle-induced osteolysis, Herbal medicine, Murine calvarial model, micro CT

Posted Date: May 25th, 2021

DOl: https://doi.org/10.21203/rs.3.rs-545163/v1

License: (9) (i) This work is licensed under a Creative Commons Attribution 4.0 International License. Read Full License 


\section{Abstract}

Background: Osteolysis is one of the most prevalent clinical complications of total joint replacement (TJR). Wedelolactone (WDL) is a coumestan compound derived from the Wedelia chinensis plant and has been demonstrated to exhibit anti-inflammatory properties. This study aimed to investigate the use of WDL as a potential treatment for reducing the risk of particle-induced osteolysis using a well-established particle-induced mice calvarial disease model.

Methods: Thirty-two C57BL/6J mice were randomized into four groups: sham, polystyrene particles (PS), PS particles with WDL treatment for 4 weeks (WDL 4w) and PS particles with WDL treatment for 8 weeks (WDL $8 \mathrm{w}$ ). Micro-CT was used to quantitatively analyze the bone mass. Osteoclast numbers were also measured from histological analysis.

Results: The results showed that bone mineral density was significantly higher in the WDL $8 \mathrm{w}$ group than in the PS group ( $p<0.05)$, and both the WDL 4 and WDL $8 \mathrm{w}$ groups had lower osteoclast numbers $(p<$ $0.05)$. No significant difference in osteoclast number was found between the WDL $4 \mathrm{w}$ and WDL $8 \mathrm{w}$ groups.

Conclusions: These results support the use of WDL as a herbal medicine for reducing the severity of particle-induced osteolysis after TJR.

\section{Background}

In clinical orthopaedic practice, artificial joint replacement (AJR) is considered an effective method for treating severe joint degeneration[1]. However, periprosthetic osteolysis resulting from the deposition of wear particles from the articulating joint is one of the major long-term complications following AJR[2]. The wear particles stimulate inflammatory responses and osteoclastic resorption processes at the bone implant interface, which consequently often leads to implant loosening $[3,4]$. Although bearing materials have been introduced to reduce the generation of wear particles, osteolysis is still prevalent and is considered a major long-term complication of AJR.

Besides changing the base material of the implant, pharmaceuticals have also been investigated as a method for reducing osteolysis [5]. Bisphosphonates are well-known drugs for treating osteoporosis, but have also been shown to be effective at suppressing osteolysis [6, 7]. Similarly, statins, which are lipidlowering agents, have been reported to reduce particle-induced osteolysis in a murine calvarial model [7]. However, such drugs can also present considerable serious side effects, such as atypical femoral bone fracture and osteonecrosis of the jaw [8-10].

Animal models are often used for investigating mechanisms that can lead to particle-induced osteolysis and for evaluating suitable treatment methods [7, 11-14]. A previous study by our institute investigated whether strontium ranelate [12], a drug for osteoporosis, could be effectively used to combat osteolysis. After gavage-feeding mice for up to 4 weeks, the results showed a significant increase in bone mineral 
density (BMD), bone volume/tissue volume (BV/TV) and trabecular thickness (Tb.Th), and a significant reduction in osteoclast numbers [12]. However, long-term use of SR presents could raise a risk of cardiovascular disease. [15-17].

As an alternative to pharmaceuticals, Chinese herbal medicines are considered a more natural solution with fewer side effects [18]. In addition, the International Organization for Standardization (ISO/TC 249) has formulated a series of standards of herbal medicines, and some studies have started to pay attention to the benefit in disease treatments [19-22]. Wedelolactone (WDL) is a coumestan compound extracted from the Wedelia Chinensis plant [23]. WDL is considered a traditional Chinese herbal medicine with strong anti-inflammatory properties [24-28]. Recent studies have shown that WDL can help promote hair growth [29] and is hepatoprotective [30, 31], neuroprotective [32] and anti-carcinogenic [33, 34].

Few studies have reported on the potential of WDL for treating diseases of the skeletal system. Based on the ability of WDL to reduce inflammation and inhibit osteoclastogenesis, it is hypothesized that WDL could also play a role in reducing the risk of particle-induced osteolysis. This study used a wellestablished murine calvarial osteolysis model to investigate whether WDL administered orally could reduce the severity of osteolysis.

\section{Methods}

Establish the calvarial particle-induced osteolysis animal model

The protocol for this experiment was approved by the Institutional Animal Care and Use Committee at the institute where the study was performed. Thirty-two 6 week old C57BL/6J female mice were supplied by BioLASCO (Taipei, Taiwan), an AAALAC certified biotechnology company. The animals were kept in a room at $24^{\circ} \mathrm{C}, 50 \%$ humidity, and with a $12 \mathrm{~h}$ light/dark cycle (light from AM 7:00 to PM 7:00). Animals were separated at random into four groups: (1) sham group $(n=8)$ (underwent surgery only) (2) vehicle group ( $n=8)$ (implanted with PS particles), (3) WDL 4w ( $=8$, PS particles and treated with WDL for 4 weeks. 7 animals remained at the end of the experiment) and (4) WDL 8w ( $n=8$, treatment for 8 weeks). Polystyrene (PS) particles have been proven to induce osteolysis (35), and so the vehicle group and WDLtreated groups were injected with $1 \mathrm{mg}$ PS particles/100 $\mu \mathrm{HA}[11,26]$. The polystyrene particles (Polystyrene Latex Spheres, 610 - 38) were purchased from TED PELLA, Inc. (CA, USA). Three hundred particles were randomly selected and measured through SEM to measure the particle size and aspect ratio (Fig. 1A). The particles were found to be $1.03 \pm 0.04 \mu \mathrm{m}$ and $0.99 \pm 0.03$, respectively (Fig. 1B, C). The particles were also tested to have an endotoxin level below $0.25 \mathrm{EU} / \mathrm{mL}$ using a Limulus Ambocyte Lysate assay kit (ToxinSensor ${ }^{\text {TM }}$ gel clot endotoxin assay kit, GenScript, NJ, USA) and then suspended in hyaluronic acid.

To implant the PS particles, the mice were anesthetized with $100 \mathrm{mg} / \mathrm{kg}$ of Zoletil 50 and $10 \mathrm{mg} / \mathrm{kg}$ Rompun by intraperitoneal injection. A $0.5 \times 0.5 \mathrm{~cm}$ area of the middle calvaria was exposed by sagittal incision. After removing the periosteum intact, the PS particle suspensions were spread over the area and the incision was closed with sutures. After 2 weeks post-surgery, the WDL 4w and 8w groups were 
gavage-fed with Wedelolactone (Y0001599, European Pharmacopoeia Reference Standard, SigmaAldrich, USA) at a dose of $4 \mathrm{mg} / \mathrm{kg} /$ day for 5 days/week [21]. WDL was dissolved by DMSO as stock solution and diluted with phosphate-buffered saline (PBS) into 10 volumes to attain a dosage of 4 $\mathrm{mg} / \mathrm{kg}$. The vehicle group was gavage-fed the vehicle solution (10\% DMSO in PBS). The sham group, vehicle group and WDL $4 \mathrm{w}$ group were then sacrificed after 4 weeks of feeding vehicle or WDL, and the WDL 8 w group was sacrificed after 8 weeks of feeding WDL.

Micro-CT imaging analysis

The calvarias were fixed in $10 \%$ buffered formalin for $24 \mathrm{~h}$, and then transferred to $70 \%$ ethanol for $24 \mathrm{~h}$. The specimens were scanned with the micro-CT system Skyscan 1076 (Bruker micro-CT, Kontich, Belgium) at a resolution of $2048 \times 2048$. Three-dimensional images were reconstructed in Skyscan system with a voxel size of $9 \mu \mathrm{m}[11,26]$. A spherical volume of interest (VOI) of $5 \mathrm{~mm}$ in diameter was then defined with the bregma as the center. Within this VOI, the bone mineral density (BMD, $\mathrm{mg} / \mathrm{cc}$ ), the ratio of bone volume to tissue volume (BV/TV, \%) and trabecular thickness ( $\mathrm{Tb}$. Th) were recorded for each group.

Histological analysis

The calvarias were decalcificated in 10\% ethylenediaminetetraacetic acid (EDTA) for 2 weeks, and paraffin embedding. Five $\mu \mathrm{m}$ thick sections of the calvaria were taken in the sagittal plane centered over the particle-treated area. The sections were then stained with hematoxylin and eosin (H\&E stain) to observe the morphology of inflammatory response of the connective tissue. A tartrate-resistant acid phosphatase (TRAP) stain was performed using a commercial TRAP kit (\#386A, Sigma-Aldrich). The number of osteoclasts was determined by counting the number of TRAP-positive multinucleated cells by two coauthors to eliminate intra- and inter-observer error.

\section{Statistical analysis}

The data were analyzed by one-way analysis of variance (ANOVA) to show the difference between groups. Multiple comparisons were adjusted with a Bonferroni post hoc test. Results were reported as mean \pm standard deviation (SD). $p$-values of less than 0.05 were considered significantly different.

\section{Results}

Micro-CT imaging analysis

A visual analysis of the three dimensional (3D) reconstructed micro-CT images showed clear differences between the sham group, vehicle group, and WDL groups (Fig. 2A). The images show typical osteolysis with pores in the sham group, but both the size and number of pores decreased in WDL-treated groups. The presence of PS particles significantly decreased the BMD in the vehicle group by $7.8 \%$ when compared to the sham group $(0.744 \pm 0.03$ for vehicle group and $0.801 \pm 0.03$ for sham group, $p<0.01)$. Both WDL groups showed an increase in BMD, with the $8 \mathrm{w}$ group showing a significant increase of $5.1 \%$ 
in comparison to the vehicle group ( $0.784 \pm 0.01$ for $8 \mathrm{w}$ group and $0.744 \pm 0.03$ for vehicle group, $p<$ 0.05). The BV/TV in the vehicle group decreased by $4.1 \%$ in comparison to the sham group $(21.23 \pm 1.43$ in vehicle group versus $22.1 \pm 1.54$ in sham group) but increased in the WDL $4 \mathrm{w}$ group $(22.96 \pm 2.07)$ and WDL $8 \mathrm{w}$ group $(22.44 \pm 2.78)$. There was no significant difference in BV/TV between the WDL $4 \mathrm{w}$ and WDL 8w. There was also no significant difference in Tb.Th between all four groups (Fig. 2B).

Histomorphometric analysis

A H\&E stain was used to evaluate the inflammatory response through histological analysis. Pseudomembrane proliferation occurred in the vehicle and WDL groups. The morphology of the cells in the periosteum was observed to change to a circle-shaped contour, while the cells resembled a flat contour in the sham group. Multinucleated giant cells were found in the surrounding periosteum (Fig. 3). TRAP staining was used to highlight PS particles in the periosteal cells and multinucleated giant cells (Fig. 4A \& B).

Osteoclasts around the bone perimeter

A TRAP stain was used to highlight osteoclasts around the calvaria in order to calculate the osteoclast numbers in each group. The results showed the osteoclast numbers in the vehicle group increased significantly in comparison to the sham group (43.74 \pm 10.09 in vehicle group versus $18.25 \pm 14.67$ in sham group, $p<0.05)$, demonstrating that the polymer particles likely induced osteolysis. Furthermore, there was a significant reduction in osteoclast numbers in the WDL $4 \mathrm{w}$ group in comparison to the vehicle group (21.09 \pm 9.79 in WDL $4 \mathrm{w}$ group versus $43.74 \pm 10.09$ in vehicle group, $p<0.05)$. No significant difference in osteoclast numbers was found between the WDL $4 \mathrm{w}$ and WDL $8 \mathrm{w}$ groups $(30.56 \pm 3.98)$ (Fig. 5).

\section{Discussion}

Osteolysis is one of the major long-term complications affecting patients following AJR. Chinese herbal medicine is considered to be milder than pharmaceutical treatments and does not produce strong adverse effects. This study aimed to investigate the potential use of the Chinese herbal medicine wedelolactone for reducing the incidence of particle-induced osteolysis.

Bisphosphonates (BPs) are commonly used to treat conditions of metabolic bone loss such as osteoporosis [36], but have also been shown to inhibit particle-induced osteolysis (37). However, the longterm use of BPs and other pharmaceuticals can often result in serious adverse effects, such as increasing the risk of osteonecrosis of the jaw, atypical femur fractures, atrial fibrillation, and esophageal cancer [38]. Statins, a drug usually used to lower blood cholesterol levels and reduce symptoms risk for illnesses related to atherosclerosis, targets the mevalonate pathway of osteoclasts, which affects the same inhibition mechanism as bisphosphonates. Statins have been shown to markedly reduce the severity of particle-induced osteolysis in a murine calvarial model [7]. However, as with BPs, the use of statins can 
present a number of side effects when used long-term, such as rhabdomyolysis, cognitive loss, neuropathy, hepatic dysfunction, and sexual dysfunction [9].

There is increasing interest in alternative methods like Traditional Chinese Medicine for treating disease as clinicians look to reduce long-term complications associated with conventional medicine. It has reported that postmenopausal Chinese women with greater fruit intake have a significantly higher BMD than comparable women with a lower fruit intake [39]. Flavonoids, found in a wide diversity of plant foods from fruits, have the most potential of dietary components for promotion of bone health than general fruits and vegetables consumption $[40,41]$. Phytoestrogens, which are natural compounds that act to maintain healthy bones, have been shown to protect against postmenopausal bone loss [20]. This protective mechanism has been demonstrated with flavones [42-44], flavanones [45], flavonols [46], coumestans [47], and triterpenoids [45, 48]. Some phytoestrogens also have the ability to reduce osteolysis by blocking some modules in the RANKL signaling pathway, and subsequently reducing the release of cytokines [42-44].

To our best knowledge, no studies to date have investigated whether WDL could reduce particle-induced osteolysis using an in-vivo mice calvarial model. The concentration of WDL in the current study was adopted from Tsai et al.[21], who showed that a low oral dose of WDL $(4 \mathrm{mg} / \mathrm{kg})$ for 4 weeks significantly suppressed the growth of prostate cancer cells. The results of our study showed that the BMD was significantly greater in the WDL $8 \mathrm{w}$ group $(0.784 \pm 0.014 \mathrm{mg} / \mathrm{cc})$ than in the vehicle group $(0.744 \pm 0.032$ $\mathrm{mg} / \mathrm{cc}$ ) (Fig. 2B). There was no significant difference between the WDL $4 \mathrm{w}$ and $8 \mathrm{w}$ groups in terms of the mean BV/TV Tb.Th, but both values were significantly less in the vehicle group. On the other hand, the osteoclast numbers were significantly lower in the WDL $4 \mathrm{w}$ group $(21.09 \pm 9.79)$ and WDL $8 \mathrm{w}$ group $(30.56 \pm 3.98)$ than the vehicle group $(44.09 \pm 9.83)$ (Fig. 5).

Previous studies treated murine calvarial osteolysis with bioactive compounds for 10 to 14 days after implantation of foreign particles [13, 42-44, 48, 49]. For instance, icariin, a bioactive flavonoid, has been proven to inhibit postmenopausal osteoporosis. Shao et al. gavage-fed mice with icariin at doses of 0.1 $\mathrm{mg} / \mathrm{g}$ and $0.3 \mathrm{mg} / \mathrm{g}$ for 14 days to examine the effects on osteolysis in a particle-induced murine calvarial model. The results showed an increase in BMD and BV/TV over the control model, and the number of TRAP positive cells decreased [13]. Similarly, ursolic acid is an abundant triterpenoid present in over one hundred species of plants. It has been reported that ursolic acid isolated from loquat leaves could reduce bone loss in OVX mice [45]. Jiang et al. treated mice with $10 \mathrm{mg} / \mathrm{kg}$ and $40 \mathrm{mg} / \mathrm{kg}$ doses of ursolic acid of intraperitoneal injection for 14 days that ursolic acid protects against wear particleinduced osteolysis by suppressing osteoclast formation and function [48]. Compared with these researches, this current study fed mice an oral dose of WDL $(4 \mathrm{mg} / \mathrm{kg})$ for 4 and 8 weeks. The treatment period in this study was longer than the other studies referenced above treating for only 2 weeks which was considered a short-term treatment. No adverse effects were observed in this study after treating the mice for 8 weeks with WDL. 
Bone remodeling is a dynamic equilibrium. Although the trigger mechanisms for osteolysis are not yet fully understood, it is known that one of the mechanisms is the receptor activation of NF-KB ligand (RANKL) and osteoprotegerin (OPG) secreted from osteoblasts and osteogenic stromal cells, both of which act to maintain the bone balance when acting normally [50]. RANKL is required for the differentiation of osteoclast precursors into mature osteoclasts [51]. As the ratio of RANKL/OPG increases, the osteoclast precursors are easier influenced by RANKL signaling through the downstream activation of NF-KB/C-fos/NFATc1, subsequently causing the precursors to differentiate into mature osteoclasts. On the other hand, macrophages also plays a key role in wear particle-induced osteolysis. Cytokines (TNF- $\alpha$ and IL-1 $\beta$, etc.) and other mediators of pro-inflammation from activated macrophages can regulate or stimulate other tissue-resident macrophages to promote osteoclastogenesis. These cytokines also regulate JNK and the p38/Erk signaling pathway to induce NFATc1, one of the downstream factors in the RANKL signaling pathway, which can lead to osteolysis.

Studies have shown that some compounds from Chinese herbal medicines can treat particle-induced osteolysis by inhibiting the modules in the NF-KB signaling pathway, the main osteolysis-related mechanism, to effect the balance of osteoclasts and osteoblasts [13,44, 48, 49]. WDL is known for its ability to block the phoporylation of $\mathrm{IKBa}$, which acts to regulate the transcription of NF-KB mediated genes, inhibiting LPS-induced pro-inflammation [49]. Annie et al. demonstrated how an extract from Wedelia chinensis attenuated Ovariectomy (OVX)-induced bone loss in mice [52]. WDL extracted from Ecliptae herba has been shown to inhibit osteoclastogenesis of RAW 264.7 cells treated with RANKL [53], and prevent OVX-induced bone loss by inhibiting osteoclast activity and enhacing osteoblast activity [54]. Furthermore, it has been confirmed WDL regulated RANKL-related NF-KB/c-fos/NFATc1 pathway can suppress osteoclastogenesis $[27,55]$, and also regulated $W n t / \beta$-catenin signaling pathway to induce osteoblastogenesis $[55,56]$. The authors concluded that oral WDL could improve bone formation and inhibit resorption by affecting the balance of osteoclasts and osteoblasts. However, the mechanism leading to the inhibition of osteolysis by WDL still needs to be determined.

Some limitations of this study should be mentioned. First, murine calvarial models allow for a low-cost study with relatively quick results, but the models use a flat bone instead of a long bone and the particles are injected on the cortical bone surface rather than in cancellous bone. Second, as detailed above, the WDL dose used in this study were adopted from other related publications. But the most effective dose for treating osteolysis in vivo has yet to be determined and requires further study. Third, osteoclast numbers were counted through qualitative analysis, not quantitative analysis. When injected onto the calvaria, the particles randomly precipitated and then a section was chosen for histological staining. This sampling approach may not represent realistic results of osteoclast numbers. Accepting the above limitations, this animal study identified the potential role of wedelolactone for treating particle-induced osteolysis.

\section{Conclusion}


This study indicated that wedelolactone (WDL), a Chinese herbal medicine, could help to maintain bone quality. Oral WDL was shown to suppress osteoclast numbers and maintain the level of BMD over time in this particle-induced osteolysis murine clavarial model. Moving forward, WDL could be potentially developed as a functional food for lowering the risk of particle-induced osteolysis after total joint replacement.

\section{Abbreviations}

Total Joint Replacement (TJR)

Wedelolactone (WDL)

Bone Mineral Density (BMD)

Bone Volume/Tissue Volume (BV/TV)

Trabecular Thickness (Tb.Th)

International Organization for Standardization (ISO)

Receptor activator of nuclear factor kappa-B ligand (RANKL)

Osteoprotegerin (OPG)

Ovariectomy (OVX)

\section{Declarations}

\section{Acknowledgments}

The authors are pleased to acknowledge the financial support of Mackay Memorial Hospital (MMH). We acknowledge experimental support from the Electron Microscope Laboratory of MMH. We also thank the Taiwan Animal Consortium (MOST 107-2319-B-001-002) Taiwan Mouse Clinic and Tron Medicalbio Corp for technical support in the micro-CT experiments. The authors particularly thank Colin J. McClean for his kind assistance in language editing and proofreading of this manuscript.

\section{Funding:}

The financial support of Mackay Memorial Hospital (MMH) and the Taiwan Animal Consortium Taiwan Mouse Clinic (MOST 107-2319-B-001-002) for the micro-CT experiments.

\section{Availability of data and materials}

The data used and/or analyzed during the current study are available from the corresponding author on reasonable request 


\section{Consent to publish}

The authors declare that they consent to publish this manuscript

\section{Authors' contributions}

Yung-Chang Lu: Conceptualization, Methodology

Tzu-Chiao Lin: Animal study, Data analysis, Writing

Ting-Kuo Chang: Methodology, Data description.

Shu-Ting Yeh: Investigation, Data analysis.

Hsu-Wei Fang: Supervision, Review \& editing.

Chun-Hsiung Huang: Supervision, Review \& editing.

Chang-Hung Huang: Supervision, Conceptualization, Writing, Review \& editing.

\section{Ethical statement}

(1)This material has not been published in whole or in part elsewhere;

(2)The manuscript is not currently being considered for publication in another journal;

(3)All authors have been personally and actively involved in substantive work leading to the manuscript, and will hold themselves jointly and individually responsible for its content.

(4)The animal protocol use in this study was approved by the Institutional Animal Care and Use Committee where the study was performed.

\section{Declaration of Competing Interest}

The authors declare that they have no known competing financial interests or personal relationships that could have appeared to influence the work reported in this paper

\section{References}

1. Harris WH. The problem is osteolysis. Clinical orthopaedics and related research. 1995(311):46-53.

2. Colston J, Atkins B. Bone and joint infection. Clin Med (Lond). 2018;18(2):150-4.

3. Silva MJ, Sandell LJ. What's new in orthopaedic research. The Journal of bone and joint surgery American volume. 2002;84-a(8):1490-6.

4. Ingham E, Fisher J. The role of macrophages in osteolysis of total joint replacement. Biomaterials. 2005;26(11):1271-86. 
5. Ren K, Dusad A, Zhang Y, Wang D. Therapeutic intervention for wear debris-induced aseptic implant loosening. Acta Pharmaceutica Sinica B. 2013;3(2):76-85.

6. O'Hara LJ, Nivbrant B, Rohrl S. Cross-linked polyethylene and bisphosphonate therapy for osteolysis in total hip arthroplasty: a case report. J Orthop Surg. 2004;12(1):114-21.

7. von Knoch F, Heckelei A, Wedemeyer C, Saxler G, Hilken G, Henschke F, et al. The effect of simvastatin on polyethylene particle-induced osteolysis. Biomaterials. 2005;26(17):3549-55.

8. Yamazaki T, Yamori M, Yamamoto K, Saito K, Asai K, Sumi E, et al. Risk of osteomyelitis of the jaw induced by oral bisphosphonates in patients taking medications for osteoporosis: a hospital-based cohort study in Japan. Bone. 2012;51(5):882-7.

9. Golomb BA, Evans MA. Statin adverse effects: a review of the literature and evidence for a mitochondrial mechanism. Am J Cardiovasc Drugs Drugs Devices Other Interv. 2008;8(6):373-418.

10. Bellosta S, Corsini A. Statin drug interactions and related adverse reactions. Exp Opin Drug Saf. 2012;11(6):933-46.

11. Huang $\mathrm{CH}$, Lu YC, Chang TK, Hsiao IL, Su YC, Yeh ST, et al. In vivo biological response to highly cross-linked and vitamin e-doped polyethylene-a particle-Induced osteolysis animal study. Journal of biomedical materials research Part B Applied biomaterials. 2016;104(3):561-7.

12. Lu YC, Chang TK, Yeh ST, Fang HW, Lin CY, Hsu LI, et al. The potential role of strontium ranelate in treating particle-induced osteolysis. Acta biomaterialia. 2015;20:147-54.

13. Shao H, Shen J, Wang M, Cui J, Wang Y, Zhu S, et al. Icariin protects against titanium particleinduced osteolysis and inflammatory response in a mouse calvarial model. Biomaterials. 2015;60:92-9.

14. Millett PJ, Allen MJ, Bostrom MP. Effects of alendronate on particle-induced osteolysis in a rat model. The Journal of bone and joint surgery American volume. 2002;84-a(2):236 - 49.

15. Reginster JY, Neuprez A, Dardenne N, Beaudart C, Emonts P, Bruyere O. Efficacy and safety of currently marketed anti-osteoporosis medications. Best Pract Res Clin Endocrinol Metab. 2014;28(6):809-34.

16. Abrahamsen B, Grove EL, Vestergaard P. Nationwide registry-based analysis of cardiovascular risk factors and adverse outcomes in patients treated with strontium ranelate. Osteoporosis international: a journal established as result of cooperation between the European Foundation for Osteoporosis and the National Osteoporosis Foundation of the USA. 2014;25(2):757-62.

17. Cooper C, Fox KM, Borer JS. Ischaemic cardiac events and use of strontium ranelate in postmenopausal osteoporosis: a nested case-control study in the CPRD. Osteoporosis international: a journal established as result of cooperation between the European Foundation for Osteoporosis and the National Osteoporosis Foundation of the USA. 2014;25(2):737 - 45.

18. Lam TP. Strengths and weaknesses of traditional Chinese medicine and Western medicine in the eyes of some Hong Kong Chinese. J Epidemiol Community Health. 2001;55(10):762.

19. Liu YQ, Wang YX, Shi NN, Han XJ, Lu AP. Current situation of International Organization for Standardization/Technical Committee 249 international standards of traditional Chinese medicine. 
Chin J Integr Med. 2017;23(5):376-80.

20. Leung PC, Siu WS. Herbal treatment for osteoporosis: a current review. J Tradit Complement Med. 2013;3(2):82-7.

21. Tsai CH, Lin FM, Yang YC, Lee MT, Cha TL, Wu GJ, et al. Herbal extract of Wedelia chinensis attenuates androgen receptor activity and orthotopic growth of prostate cancer in nude mice. Clin Cancer Res. 2009;15(17):5435-44.

22. Tang XF, Ma ZT, Gao YY, Wang H, Li XX, Yu P, et al. Systemic osteoprotective effects of Epimedii Folium and Ligustri Lucidi Fructus in senile osteoporosis rats by promoting the osteoblastogenesis and osteoclastogenesis based on MLP-ANN model. Chin Med. 2020;15:87.

23. Govindachari TR, Nagarajan K, Parthasarathy PC. Synthetic analogues of wedelolactone. Tetrahedron. 1961;15(1):129-31.

24. Huang YT, Wen CC, Chen YH, Huang WC, Huang LT, Lin WC, et al. Dietary uptake of Wedelia chinensis extract attenuates dextran sulfate sodium-induced colitis in mice. PloS one. 2013;8(5):e64152-e.

25. Lin WC, Wen CC, Chen YH, Hsiao PW, Liao JW, Peng Cl, et al. Integrative approach to analyze biodiversity and anti-inflammatory bioactivity of Wedelia medicinal plants. PloS one. 2015;10(6):e0129067-e.

26. Ding S, Hou X, Yuan J, Tan X, Chen J, Yang N, et al. Wedelolactone protects human bronchial epithelial cell injury against cigarette smoke extract-induced oxidant stress and inflammation responses through Nrf2 pathway. Int Immunopharmacol. 2015;29(2):648-55.

27. Yuan F, Chen J, Sun PP, Guan S, Xu J. Wedelolactone inhibits LPS-induced pro-inflammation via NFkappaB pathway in RAW 264.7 cells. Journal of biomedical science. 2013;20:84.

28. Kobori M, Yang Z, Gong D, Heissmeyer V, Zhu H, Jung YK, et al. Wedelolactone suppresses LPSinduced caspase-11 expression by directly inhibiting the IKK complex. Cell death differentiation. 2004;11(1):123-30.

29. Roy RK, Thakur M, Dixit VK. Hair growth promoting activity of Eclipta alba in male albino rats. Arch Dermatol Res. 2008;300(7):357-64.

30. Lu Y, Hu D, Ma S, Zhao X, Wang S, Wei G, et al. Protective effect of wedelolactone against CCI4induced acute liver injury in mice. Int Immunopharmacol. 2016;34:44-52.

31. Luo Q, Ding J, Zhu L, Chen F, Xu L. Hepatoprotective Effect of Wedelolactone against Concanavalin A-Induced Liver Injury in Mice. Am J Chin Med. 2018;46(4):819-33.

32. Maya S, Prakash T, Goli D. Evaluation of neuroprotective effects of wedelolactone and gallic acid on aluminium-induced neurodegeneration: Relevance to sporadic amyotrophic lateral sclerosis. Eur $\mathrm{J}$ Pharmacol. 2018;835:41-51.

33. Peng YG, Zhang L. Wedelolactone suppresses cell proliferation and migration through AKT and AMPK signaling in melanoma. The Journal of dermatological treatment. 2018:1-7.

34. Nehybova T, Smarda J, Daniel L, Stiborek M, Kanicky V, Spasojevic I, et al. Wedelolactone Acts as Proteasome Inhibitor in Breast Cancer Cells. International journal of molecular sciences. 2017;18(4). 
35. Ma T, Ortiz SG, Huang Z, Ren P, Smith RL, Goodman SB. In vivo murine model of continuous intramedullary infusion of particles-a preliminary study. Journal of biomedical materials research Part B Applied biomaterials. 2009;88(1):250-3.

36. Seedor JG, Quartuccio HA, Thompson DD. The bisphosphonate alendronate (MK-217) inhibits bone loss due to ovariectomy in rats. Journal of bone mineral research: the official journal of the American Society for Bone Mineral Research. 1991;6(4):339-46.

37. Purdue PE, Koulouvaris P, Potter HG, Nestor BJ, Sculco TP. The cellular and molecular biology of periprosthetic osteolysis. Clin Orthop Relat Res. 2007;454:251-61.

38. McClung M, Harris ST, Miller PD, Bauer DC, Davison KS, Dian L, et al. Bisphosphonate therapy for osteoporosis: benefits, risks, and drug holiday. Am J Med. 2013;126(1):13-20.

39. Chen YM, Ho SC, Woo JL. Greater fruit and vegetable intake is associated with increased bone mass among postmenopausal Chinese women. Br J Nutr. 2006;96(4):745-51.

40. Weaver CM, Alekel DL, Ward WE, Ronis MJ. Flavonoid intake and bone health. J Nutr Gerontol Geriatr. 2012;31(3):239-53.

41. Welch AA, Hardcastle AC. The effects of flavonoids on bone. Curr Osteoporos Rep. 2014;12(2):20510.

42. Yu X, Zhao X, Wu T, Zhou Z, Gao Y, Wang X, et al. Inhibiting wear particles-induced osteolysis with naringin. Int Orthop. 2013;37(1):137-43.

43. Shin DK, Kim MH, Lee SH, Kim TH, Kim SY. Inhibitory effects of luteolin on titanium particle-induced osteolysis in a mouse model. Acta biomaterialia. 2012;8(9):3524-31.

44. Zhao S, Sun Y, Li X, Wang J, Yan L, Zhang Z, et al. Scutellarin inhibits RANKL-mediated osteoclastogenesis and titanium particle-induced osteolysis via suppression of NF-kappaB and MAPK signaling pathway. Int Immunopharmacol. 2016;40:458-65.

45. Wang W, Wu C, Tian B, Liu X, Zhai Z, Qu X, et al. The inhibition of RANKL-induced osteoclastogenesis through the suppression of $\mathrm{p} 38$ signaling pathway by naringenin and attenuation of titaniumparticle-induced osteolysis. Int J Mol Sci. 2014;15(12):21913-34.

46. Wang QS, Wang GF, Lu YR, Cui YL, Li H, Li RX, et al. The Combination of icariin and constrained dynamic loading stimulation attenuates bone loss in ovariectomy-induced osteoporotic mice. J Orthop Res. 2018;36(5):1415-24.

47. Zhai Y, Li Y, Wang Y, Cui J, Feng K, Kong X, et al. Psoralidin, a prenylated coumestan, as a novel antiosteoporosis candidate to enhance bone formation of osteoblasts and decrease bone resorption of osteoclasts. Eur J Pharmacol. 2017;801:62-71.

48. Jiang C, Xiao F, Gu X, Zhai Z, Liu X, Wang W, et al. Inhibitory effects of ursolic acid on osteoclastogenesis and titanium particle-induced osteolysis are mediated primarily via suppression of NF-kappaB signaling. Biochimie. 2015;111:107-18.

49. Jiao Z, Xu W, Zheng J, Shen P, Qin A, Zhang S, et al. Kaempferide Prevents Titanium Particle Induced Osteolysis by Suppressing JNK Activation during Osteoclast Formation. Scientific reports. 2017;7(1):16665. 
50. Boyce BF, Xing L. Biology of RANK, RANKL, and osteoprotegerin. Arthritis research therapy. 2007;9(Suppl 1):1.

51. Teitelbaum SL, Ross FP. Genetic regulation of osteoclast development and function. Nature reviews Genetics. 2003;4(8):638-49.

52. Annie S, Prabhu RG, Malini S. Activity of Wedelia calendulacea Less. in post-menopausal osteoporosis. Phytomedicine: international journal of phytotherapy phytopharmacology. 2006;13(12):43-8.

53. Liu YQ, Zhan LB, Liu T, Cheng MC, Liu XY, Xiao HB. Inhibitory effect of Ecliptae herba extract and its component wedelolactone on pre-osteoclastic proliferation and differentiation. J Ethnopharmacol. 2014;157:206-11.

54. Liu YQ, Hong ZL, Zhan LB, Chu HY, Zhang XZ, Li GH. Wedelolactone enhances osteoblastogenesis by regulating Wnt/beta-catenin signaling pathway but suppresses osteoclastogenesis by NF-kappaB/cfos/NFATc1 pathway. Sci Rep. 2016;6:32260.

55. Deng X, Liang L-N, Zhu D, Zheng L-P, Yu J-H, Meng X-I, et al. Wedelolactone inhibits osteoclastogenesis but enhances osteoblastogenesis through altering different semaphorins production. Int Immunopharmacol. 2018;60:41-9.

56. Zhu D, Deng X, Han XF, Sun XX, Pan TW, Zheng LP, et al. Wedelolactone Enhances Osteoblastogenesis through ERK- and JNK-mediated BMP2 Expression and Smad/1/5/8 Phosphorylation. Molecules. 2018;23(3):561.

\section{Figures}


(A)

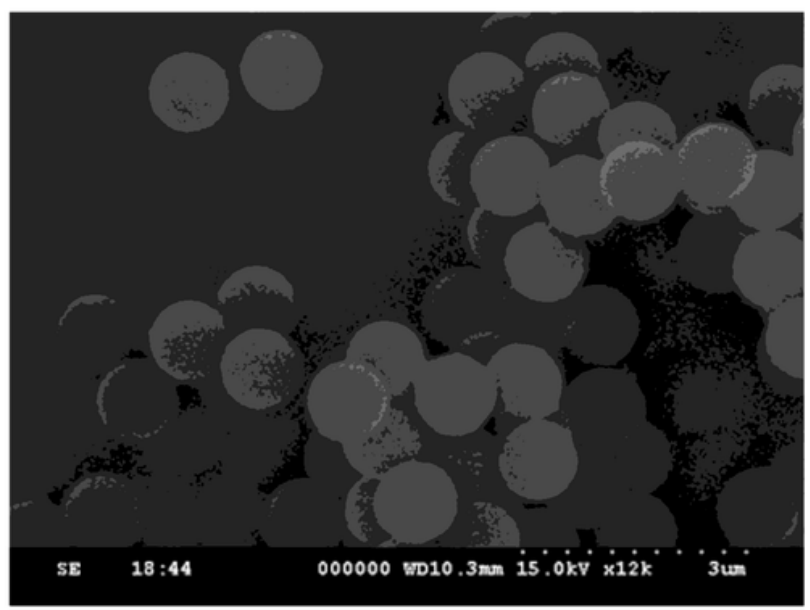

(C)

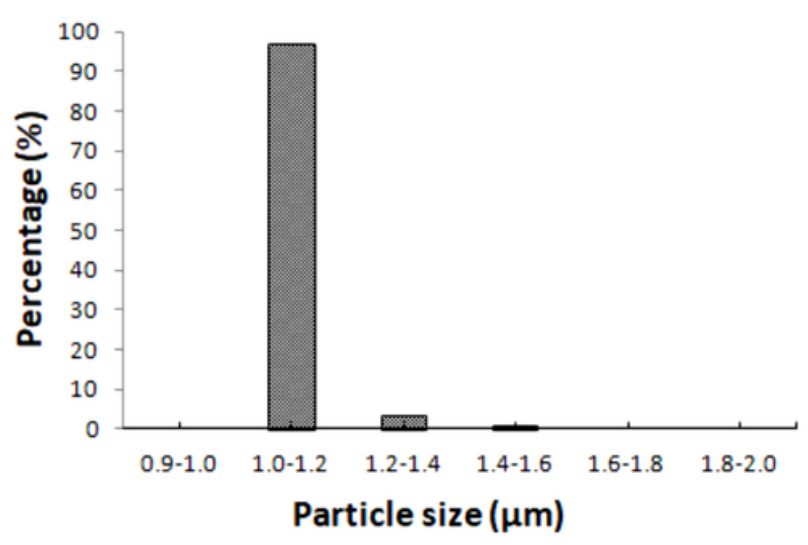

(B)

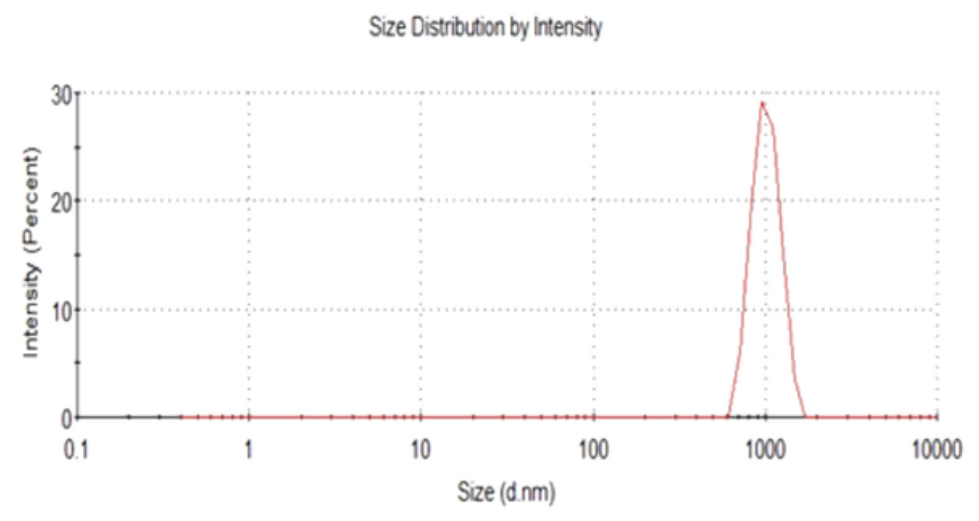

(D)

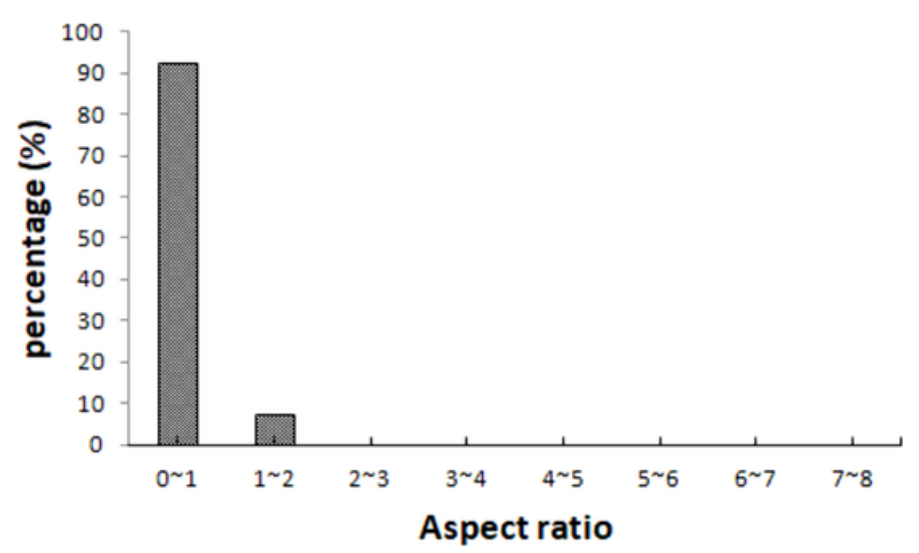

\section{Figure 1}

SEM image $(\times 12,000)$ of polystyrene (PS) particles $(A)$. The particle size distribution by light-scattering analysis (B). The particle size distribution (C) and the aspect ratio (D) of the particles from SEM images. 
(A)
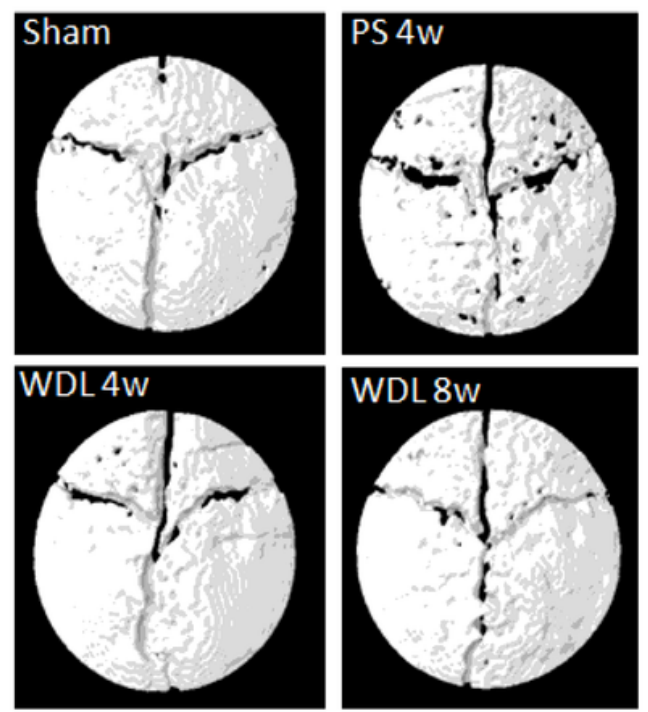

WDL 8w

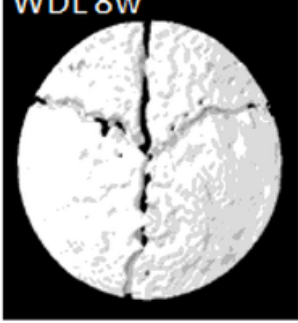

(B)
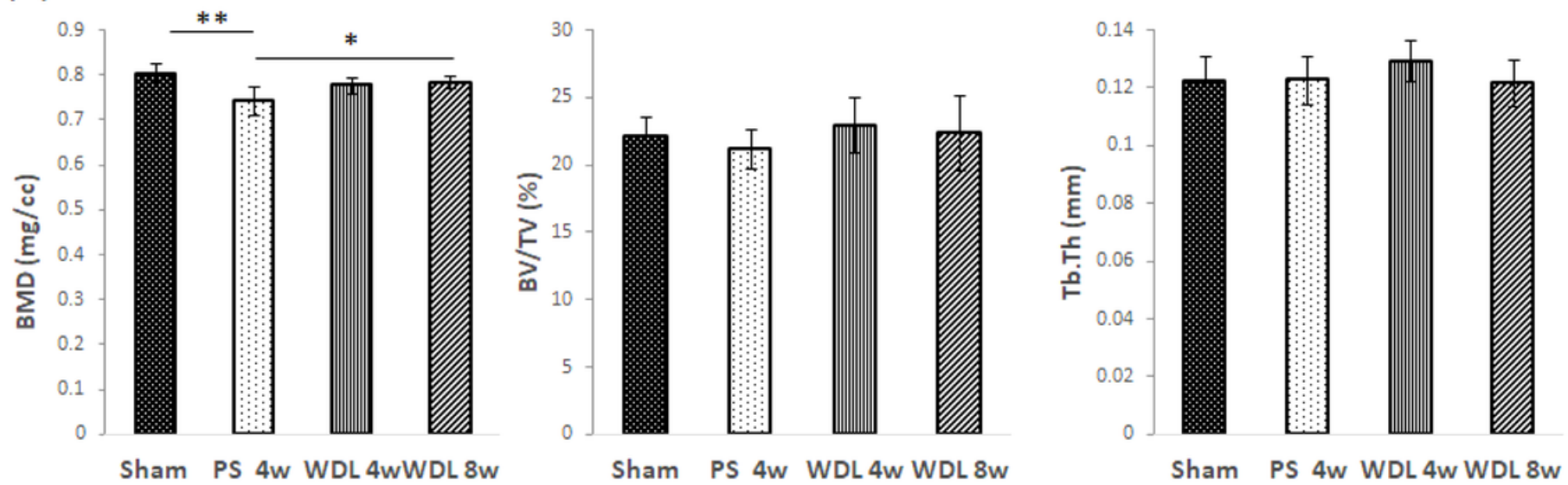

Figure 2

Reconstructed image of the VOI with the bregma at the center. The VOI is defined with a diameter of 5 $\mathrm{mm}$ (A). Micro-CT image of bone formation in a particle-induced osteolysis model measured at 4 and 8 weeks after feeding WDL (B) $\left({ }^{*} p<0.05 ; * \star p<0.01\right.$, as determined using ANOVA testing). 


\section{Sham}

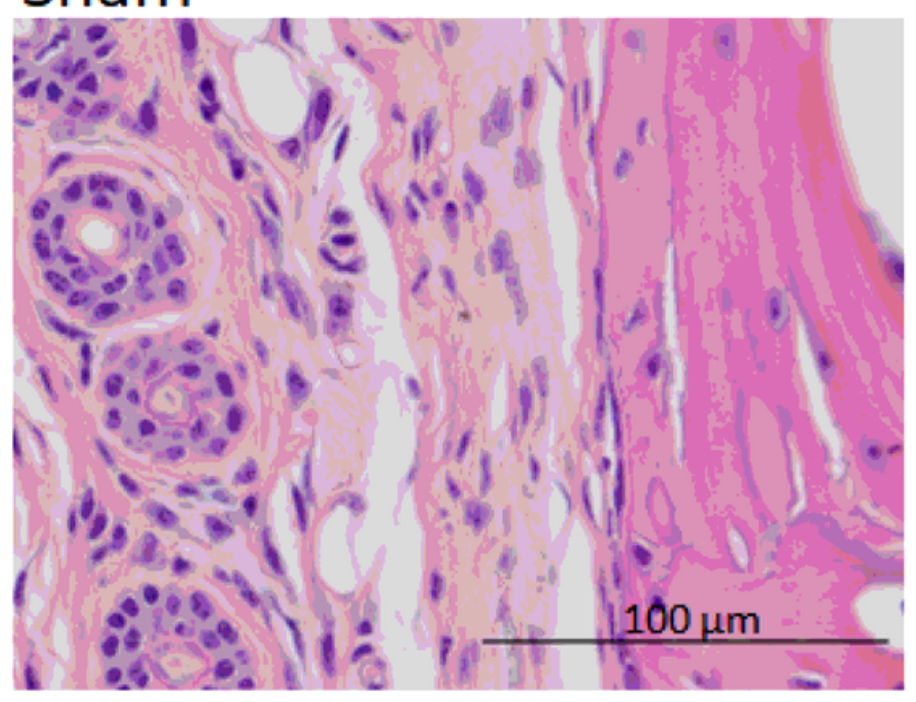

\section{WDL 4W}

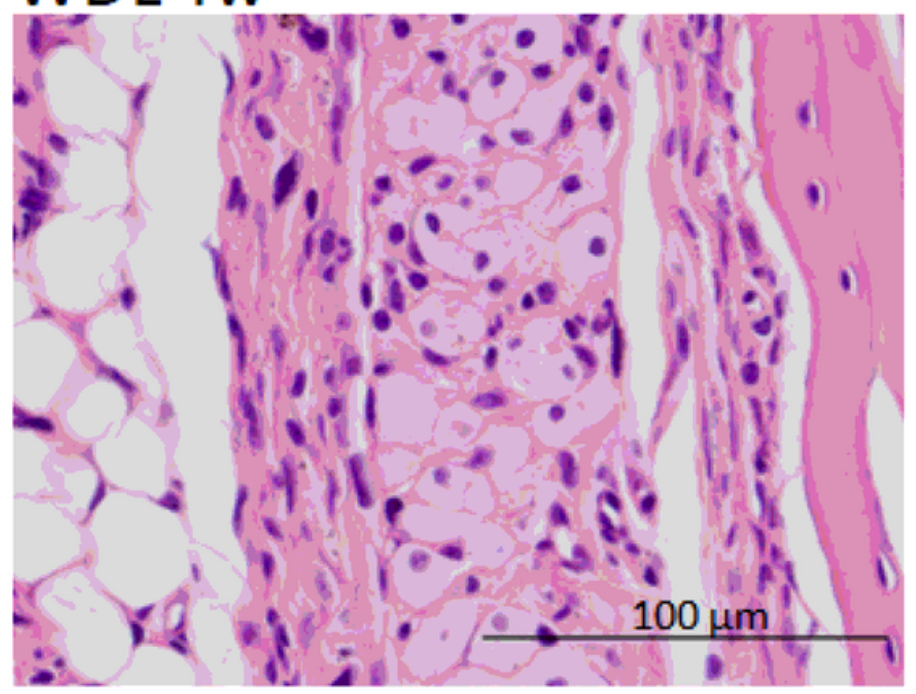

PS 4W

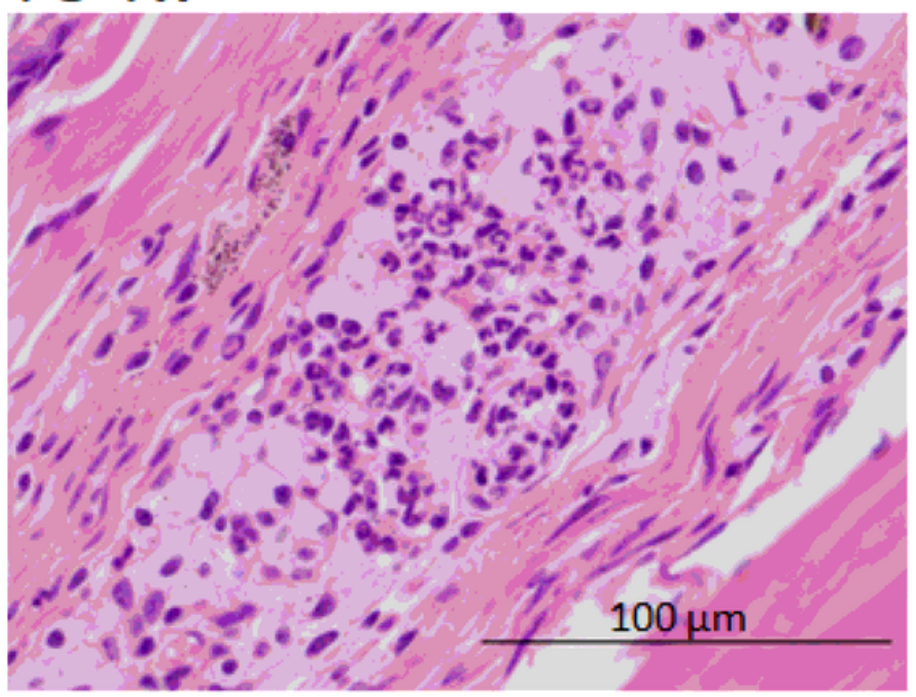

\section{WDL $8 \mathrm{~W}$}

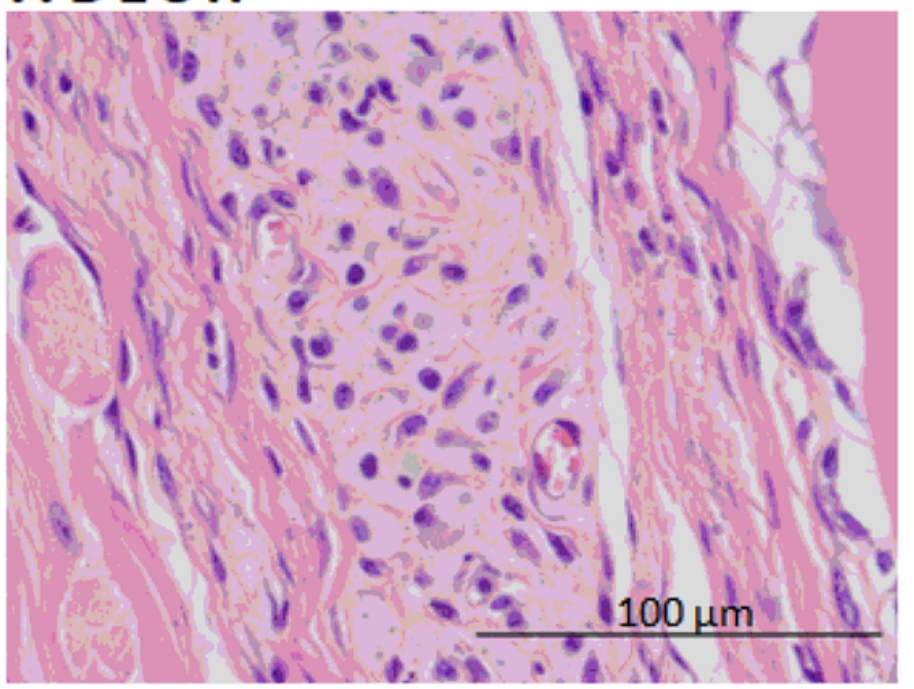

\section{Figure 3}

Hematoxylin and eosin (H\&E) staining of periosteum in mice calvarial section. Multinucleated giant cells were observed in the groups injected with PS particles (Magnification: × 40; scale bar: $100 \mu \mathrm{m}$ ). 
(A)

Sham
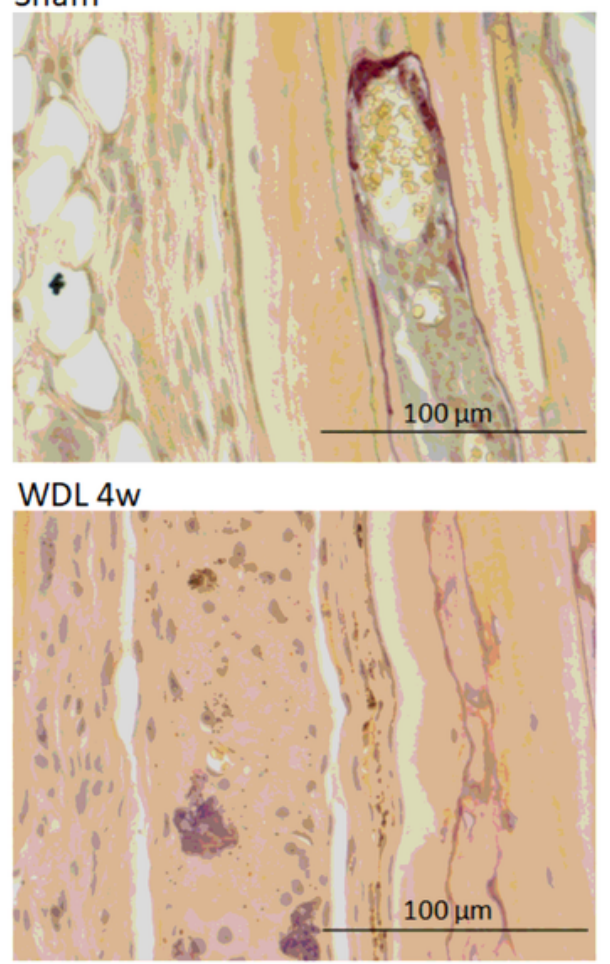

PS $4 w$

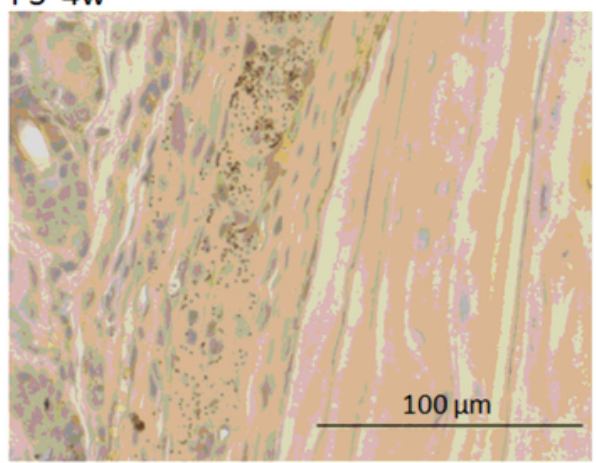

WDL $8 \mathrm{~W}$

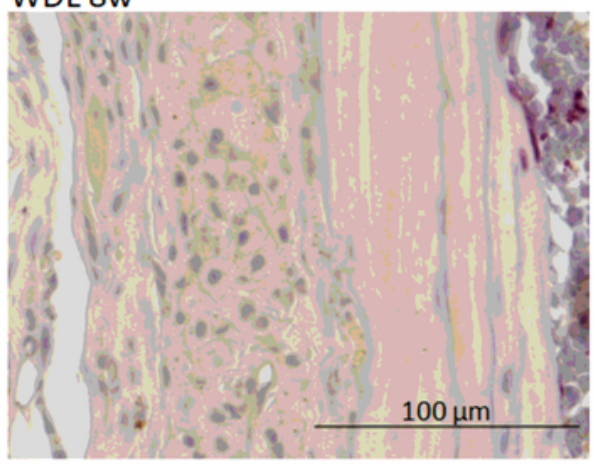

(B)

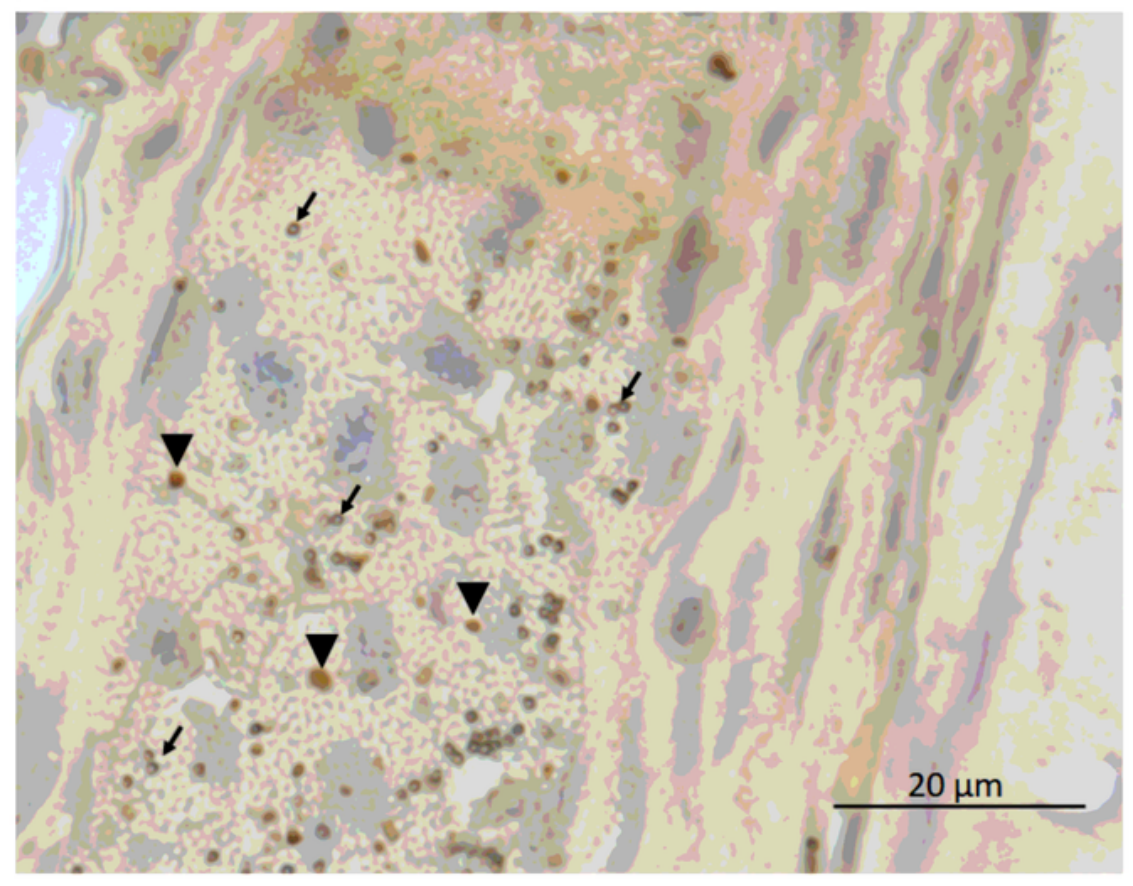

Figure 4

TRAP staining indicated that PS particles exist in the periosteal cells and multinucleated giant cells in mice calvarial tissue $(A)$ (Magnification $\times 40$ Scale bar: $100 \mu \mathrm{m}$ ). Different shapes of PS particles and melanin granules observed in TRAP staining. Arrow, PS particle; Arrow head, melanin granule (B) (Magnification $\times 100$ Scale bar: $20 \mu \mathrm{m}$ ). 
(A)
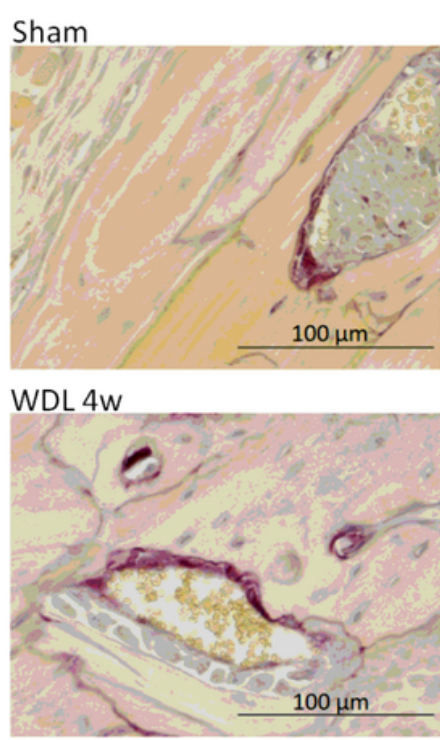

PS 4 w

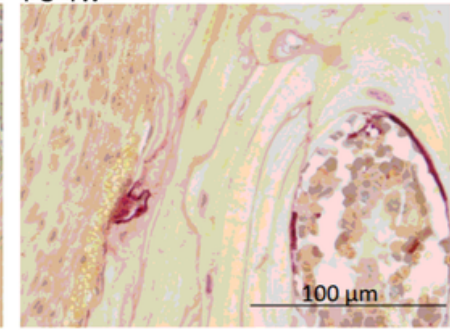

WDL 8 W

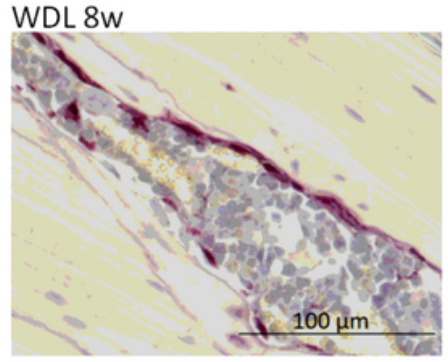

(B)

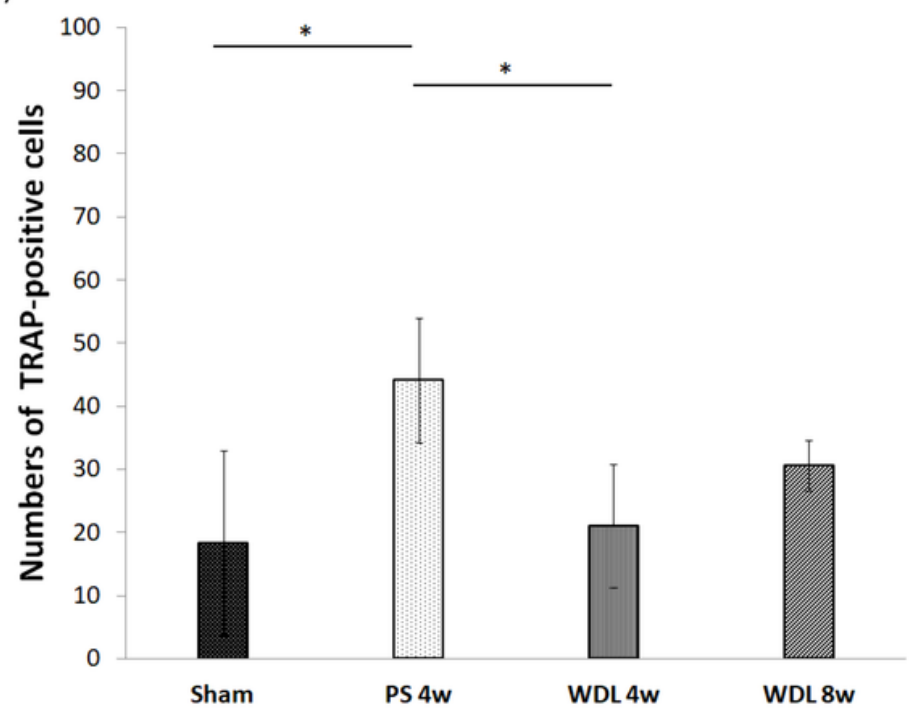

Figure 5

Typical samples from micro-CT with purple staining showing TRAP-positive osteoclasts (A) (Magnification: $\times 40$; Scale bar: $100 \mu \mathrm{m}$ ). Average number of TRAP-positive cells from each group are presented as the mean $\pm S D(B)\left({ }^{*} p<0.05\right)$. 